\title{
RESULTADOS DE LA DACRIOCISTORRINOSTOMÍA EXTERNA VÍA INFERIOR REALIZADA POR MÉDICOS RESIDENTES
}

\author{
OUTCOMES OF INFERIOR ACCESS EXTERNAL \\ DACRYOCYSTORHINOSTOMY PERFORMED BY RESIDENTS
}

\author{
MASCARÓ-ZAMORA F ${ }^{1}$, TORIBIO-GARCIA JA², BARBERÁ-LOUSTAUNAU E ${ }^{3}$
}

\begin{abstract}
RESUMEN
Objetivo: Analizar los resultados de la dacriocistorrinostomía externa (DCREx) realizada por los médicos residentes del Hospital Universitari de Bellvitge, estudiando las diferencias de sus cirugías respecto a los médicos adjuntos.

Métodos: Realizamos un estudio retrospectivo estudiando todas las DCREx realizadas entre enero de 2006 y diciembre de 2007 en nuestro centro que correspondieron a 65 cirugías de 54 pacientes, de las cuales 16 fueron realizadas por médicos residentes y 49 por los médicos adjuntos.

Se constituyen dos grupos: casos intervenidos por los médicos residentes y casos intervenidos por los médicos adjuntos, y se estudian las siguientes variables: características epidemiológicas de los pacientes intervenidos (edad, sexo), lado de intervención, antecedente de inflamación del saco lagrimal, tipo de anestesia empleada, complicaciones intraquirúrgicas, postquirúrgicas y resultados de la cirugía. Se define como «éxito anatómico» cuando se comprueba la permeabilidad de la vía lagrimal al irrigar suero, y «éxito funcional» cuando además el paciente no refiere síntomas de epífora.
\end{abstract}

Resultados: En el 88\% de los casos se obtuvieron buenos resultados: en un $80 \%$ de los casos se alcan-

\begin{abstract}
Purpose: To assess the outcomes of external dacryocystorhinostomy (DCREx) performed by residents at the Hospital Universitari de Bellvitge, comparing the cases operated on by residents and the cases operated on by staff surgeons.

Methods: We performed a retrospective study between January 2006 and December 2007 of all DCREx performed at our hospital. The operations involved 65 cases from 54 patients: 16 operations were performed by residents and 49 by staff surgeons.

The cases were divided into two groups: cases operated on by residents and cases operated on by staff surgeons, and the following variables were studied: epidemiologic characteristics of the patients (age, sex), side of intervention, antecedents of lacrimal sac inflammation, type of anesthesia, intrasurgical and postsurgical complications and results. «Anatomic success» was defined as the possibility to pass physiologic serum through the lacrimal pathway and «functional success» if the patient in addition was without symptoms of epiphora.
\end{abstract}

Results: The procedure was successful in $88 \%$ of cases: $80 \%$ were classified as «functional success» while only $8 \%$ were considered «anatomic success». The operations performed by residents were

\footnotetext{
Recibido: 18/8/08. Aceptado: 26/10/09.

Hospital Universitari de Bellvitge. Barcelona. España.

${ }^{1}$ Licenciado en Medicina. Unidad de Órbita. Hospital Universitari de Bellvitge. Barcelona. España.

2 Licenciado en Medicina. Complejo Asistencial de León. León. España.

${ }^{3}$ Licenciado en Medicina. Complejo Hospitalario Universitario Juan Canalejo de La Coruña. La Coruña. España.

Trabajo presentado parcialmente en el XVII Congreso de la Sociedad Española de Cirugía Plástica Ocular y Orbitaria (Valencia 2007).

Correspondencia:

J. Álvaro Toribio García

Avda. Compostilla, 24 - 3. ${ }^{\circ} \mathrm{B}$

24400 Ponferrada (León)

España

E-mail: draltor@gmail.com
} 
zó éxito funcional y en un $8 \%$ sólo anatómico. En las cirugías realizadas por los médicos residentes se consiguió un éxito global del 81\% (75\% funcional-6\% anatómico), frente al $90 \%$ de éxito global alcanzado por los adjuntos ( $82 \%$ funcional-8\% anatómico).

Conclusión: Los resultados indican que con el adecuado entrenamiento y supervisión, en la DCREx los residentes pueden obtener tasas de éxito satisfactorias, muy próximas a las alcanzadas por los adjuntos, sin que exista un incremento en las complicaciones.

Palabras clave: Dacriocistorrinostomía externa, vía inferior, médicos residentes, médicos adjuntos, resultados, complicaciones. successful in $81 \%$ of cases $(75 \%$ «functional success» and 6\% «anatomic success»), whereas staff surgeons achieved success in $90 \%$ of cases $(82 \%$ «functional success» $-8 \%$ «anatomic success»).

Conclusion: The results show that residents with suitable training and supervision can achieve good success rates approaching those of staff surgeon outcomes, without additional complications (Arch Soc Esp Oftalmol 2009; 84: 501-506).

Key Words: External dacryocystorhinostomy, inferior access, residents, staff surgeons, results, complications.

\section{INTRODUCCIÓN}

La dacriocistorrinostomía externa (DCREx) continúa siendo el tratamiento más efectivo para resolver la obstrucción de vía lagrimal baja (OVLB) en adultos, que fundamentalmente se produce por estenosis del conducto nasolagrimal $(1,2)$. Esta técnica pretende crear una comunicación directa entre la mucosa del saco lagrimal y la mucosa de la fosa nasal, a través de una osteotomía practicada en la foseta lagrimal $(3,4)$. Es un procedimiento eficaz con una alta tasa de éxito (80-99\% según el estudio (4)), aunque no es una técnica sencilla y requiere una considerable habilidad quirúrgica. Además, la experiencia del cirujano parece ser un factor decisivo a la hora de alcanzar buenos resultados (2).

El objetivo de nuestro trabajo es analizar las DCREx realizadas por médicos residentes bajo supervisión, estudiando las diferencias de sus cirugías respecto a los médicos adjuntos en cuanto a complicaciones y resultados. Asimismo estudiaremos las características epidemiológicas de los pacientes intervenidos y valoraremos algunos factores que se asocian con un mayor índice de fracaso.

\section{SUJETOS, MATERIAL Y MÉTODOS}

Se realizó un estudio retrospectivo estudiando todas las DCREx realizadas entre enero de 2006 y diciembre de 2007 que correspondieron a 65 cirugías de 54 pacientes, de las cuales 16 fueron realizadas por médicos residentes y 49 por los médicos adjuntos. Los médicos residentes en nuestro hospital realizan una estancia de 6 meses en la unidad de órbita, independientemente de su año de residencia. Después de los dos primeros meses comienzan a realizar DCREx supervisados por los adjuntos.

El diagnóstico que motivó la realización de la DCREx fue en todos los casos de OVLB. Este diagnóstico se realizó de forma clínica en todos los pacientes comprobando la permeabilidad de la vía lagrimal alta (sondando el canalículo inferior hasta el saco lagrimal) y la OVLB al irrigar con suero y no alcanzar éste la faringe. Se clasificó la OVLB en función del grado clínico de inflamación, interrogando a los pacientes por el antecedente de dacriocistitis (OVLB con dacriocistitis), y si éste era negativo valorando si a la palpación el saco estaba dilatado y refluía material mucoso al presionar (OVLB con mucocele) o si el suero refluía limpio y no existía dilatación (OVLB simple).

No fueron incluidos en el estudio los pacientes con OVLB en los que se realizó otra técnica quirúrgica, como la dacriocistorrinostomía endonasal o la dacriocistorrinostomía transcalicular con láser.

Después de obtener el consentimiento informado verbal y escrito por parte del paciente y pasar a lista de espera quirúrgica, se realizó la DCREx por vía inferior. En esta variante de la técnica original, se emplea el pliegue palpebral inferior como vía de acceso indirecto al saco lagrimal, al contrario que en DCREx clásica en la que la incisión se realiza sobre la piel que cubre el tendón cantal medial, obteniendo tras retirar éste un acceso directo al saco lagrimal. La DCREx por vía inferior presenta el 
inconveniente de realizar un trayecto más largo hasta alcanzar la pared ósea que debemos trepanar, pero presenta la ventaja estética de disimular la cicatriz en el pliegue palpebral inferior.

Se comenzó la operación colocando de 3 a 6 lentinas de gasa empapadas en Tetracaína al $1 \%$ y Epinefrina $1 / 100.000$ en la fosa nasal a intervenir durante unos 15 minutos. Se realizó la incisión siguiendo el pliegue palpebral inferior y se disecó el músculo orbicular hasta llegar al reborde orbitario. Después de cortar el periostio con el bisturí eléctrico se despegó del hueso hasta alcanzar la foseta lagrimal. La osteotomía se hizo sobre la cresta lagrimal anterior, perteneciente a la apófisis ascendente del hueso maxilar, y se incidió la mucosa nasal elaborando un colgajo. Después de localizar el saco lagrimal se abrió, extirpando la pared posterior y las posibles tabicaciones intrasacales. Se realizó un sondaje bicanalicular con tubos de silicona pasándolos a través de la osteotomía a la fosa nasal, y se suturó con dos puntos de material reabsorvible la pared anterior del saco lagrimal al colgajo de mucosa nasal. El cierre por planos de músculo orbicular y piel se hizo con puntos simples.

Tras la cirugía se prescribió a los pacientes tratamiento sistémico con antibiótico y corticoides durante 7 días, y colirio antibiótico-corticoideo cuatro veces al día durante una semana disminuyendo el número de aplicaciones de forma progresiva hasta su retirada a los 30 días. Se evaluó a los pacientes a la semana, al primer mes y al tercer mes en todos los casos, realizando irrigación de la vía lagrimal e interrogando por la ausencia subjetiva de epífora. Los tubos de silicona se retiraron a las cinco semanas de la intervención. El seguimiento medio fue de 6,2 meses (4-12 meses).

Se establecieron dos grupos: pacientes intervenidos por los médicos adjuntos y pacientes intervenidos por los médicos residentes, y se estudiaron las siguientes variables: características epidemiológicas de los pacientes intervenidos (edad, sexo), lado de intervención, inflamación clínica del saco lagrimal, tipo de anestesia empleada, complicaciones intraquirúrgicas (sangrado, trepanación, intubación y sutura de mucosas), complicaciones postquirúrgicas (enfisema, epistaxis, queloide y salida incompleta de tubos de silicona) y resultados de la cirugía. Los resultados se clasificaron como «éxito anatómico» cuando existía permeabilidad de la vía lagrimal al irrigar con suero, y «éxito funcional» cuando además el paciente no refería síntomas de epífora.
También se valoró si, independientemente del grado de experiencia del cirujano, existía alguna asociación entre las variables a estudio y el resultado quirúrgico.

Todos los datos fueron procesados usando el programa SPSS 13.0 para Windows (SPSS for Windows, SPSS Inc, Chicago, USA). Los estadísticos aplicados fueron el test de Chi-cuadrado $\left(\mathrm{X}^{2}\right)$ y el test $\mathrm{T}$ de Student, con un nivel de confianza del $95 \%$.

\section{RESULTADOS}

En cuanto a las características epidemiológicas de los pacientes intervenidos, la edad media fue de 66 años (SD: 11; rango: 40-88), 45 pacientes (69\%) eran mujeres y 20 eran varones (31\%). De los 54 pacientes analizados la cirugía fue bilateral en 11, totalizando 65 procedimientos. De las intervenciones realizadas 31 se desarrollaron sobre el lado derecho (48\%) y 34 sobre el lado izquierdo (52\%). En cuanto al grado clínico de inflamación, 21 $(32 \%)$ de las intervenciones se clasificaron como OVLB con dacriocistitis, 30 (46\%) de OVLB con mucocele y 14 (22\%) de OVLB simple. Las intervenciones realizadas por los médicos adjuntos fueron $49(75 \%)$ y las realizadas por los médicos residentes 16 (25\%), siendo sus características epidemiológicas y de inflamación del saco lagrimal similares (tabla I).

Respecto a las características quirúrgicas, la anestesia empleada fue local en $34(52 \%)$ de las cirugías y general en 31 (48\%) de las intervenciones. Sin embargo existen diferencias estadísticamente significativas $(\mathrm{p}=0,012)$ entre el grupo de los médicos adjuntos (emplearon anestesia local en 30 $(61 \%)$ de sus intervenciones) y el grupo de los médicos residentes (emplearon anestesia local sólo en $4(25 \%)$ de sus intervenciones). En cuanto a las complicaciones intraquirúrgicas, la más relevante fue el sangrado produciéndose en 35 (54\%) de las intervenciones. Clasificamos el sangrado en tres grados: grado 1 se produce sangrado pero no se modifica la técnica quirúrgica; grado 2: se produce sangrado que obliga a modificar la técnica quirúrgica; y grado 3: sangrado severo que obliga a terminar la intervención con un taponamiento nasal. El sangrado severo sólo se produjo en 7 (11\%) de las intervenciones, sin diferencias entre adjuntos y residentes. Tanto la trepanación ósea como la intuba- 
Tabla. I. Frecuencias observadas respecto a las características de los pacientes y a las características de la cirugía, según el tipo de médico que realiza las intervenciones quirúrgicas (IQ)

\begin{tabular}{|c|c|c|c|c|c|c|c|}
\hline \multirow[b]{2}{*}{ Intervenciones DCREx (n) } & \multicolumn{2}{|c|}{ Global } & \multicolumn{2}{|c|}{$\begin{array}{c}\text { Grupo I } \\
\text { IQ. Adjuntos }\end{array}$} & \multicolumn{2}{|c|}{$\begin{array}{c}\text { Grupo II } \\
\text { IQ. Residentes }\end{array}$} & \multirow[t]{2}{*}{$\begin{array}{c}\text { Estadístico } \\
\text { y p-valor }\end{array}$} \\
\hline & 65 & $100 \%$ & 49 & $75 \%$ & 16 & $25 \%$ & \\
\hline Edad & \multicolumn{2}{|c|}{66 DE 11} & \multicolumn{2}{|c|}{66 DE 11} & \multicolumn{2}{|c|}{$66 \mathrm{DE} 12$} & $\begin{array}{c}\text { T de Student } \\
\mathrm{p}=0,950\end{array}$ \\
\hline \multicolumn{8}{|l|}{ Sexo } \\
\hline Varones & 20 & $31 \%$ & 15 & $31 \%$ & 5 & $31 \%$ & \\
\hline Mujeres & 45 & $69 \%$ & 34 & $69 \%$ & 11 & $69 \%$ & $X^{2}, p=0,962$ \\
\hline \multicolumn{8}{|l|}{ Lado } \\
\hline Derecho & 31 & $48 \%$ & 24 & $49 \%$ & 7 & $44 \%$ & \\
\hline Izquierdo & 34 & $52 \%$ & 25 & $51 \%$ & 9 & $56 \%$ & $\mathrm{X}^{2}, \mathrm{p}=0,716$ \\
\hline \multicolumn{8}{|l|}{ Inflamación } \\
\hline OVLB + Dacriocistitis & 21 & $32 \%$ & 14 & $29 \%$ & 7 & $44 \%$ & \\
\hline OVLB + Mucocele & 30 & $46 \%$ & 25 & $51 \%$ & 5 & $31 \%$ & \\
\hline OVLB + Simple & 14 & $22 \%$ & 10 & $20 \%$ & 4 & $25 \%$ & $X^{2}, p=0,368$ \\
\hline \multicolumn{8}{|l|}{ Anestesia } \\
\hline Local & 34 & $52 \%$ & 30 & $61 \%$ & 4 & $25 \%$ & \\
\hline General & 31 & $48 \%$ & 19 & $39 \%$ & 12 & $75 \%$ & $X^{2}, p=0,012$ \\
\hline \multicolumn{8}{|l|}{ Complicaciones intraquirúrgicas } \\
\hline Sangrado (Grado 1,2 ó 3) & 35 & $54 \%$ & 26 & $53 \%$ & 9 & $56 \%$ & $\mathrm{X}^{2}, \mathrm{p}=0,748$ \\
\hline Sangrado severo (Grado 3) & 7 & $11 \%$ & 5 & $10 \%$ & 2 & $13 \%$ & $\mathrm{X}^{2}, \mathrm{p}=0,797$ \\
\hline Trepanación defectuosa & 0 & $0 \%$ & 0 & $0 \%$ & 0 & $0 \%$ & \\
\hline Intubación defectuosa & 0 & $0 \%$ & 0 & $0 \%$ & 0 & $0 \%$ & \\
\hline Sutura mucosa 1 punto & 23 & $35 \%$ & 18 & $37 \%$ & 5 & $31 \%$ & $X^{2}, p=0,690$ \\
\hline \multicolumn{8}{|l|}{ Complicaciones postquirúrgicas } \\
\hline Enfisema & 0 & $0 \%$ & 0 & $0 \%$ & 0 & $0 \%$ & \\
\hline Epixtasis & 6 & $9 \%$ & 6 & $12 \%$ & 0 & $0 \%$ & $X^{2}, p=0,142$ \\
\hline Queloide & 4 & $6 \%$ & 3 & $6 \%$ & 1 & $6 \%$ & $\mathrm{X}^{2}, \mathrm{p}=0,985$ \\
\hline Salida incompleta tubos & 13 & $20 \%$ & 10 & $20 \%$ & 3 & $19 \%$ & $\mathrm{X}^{2}, \mathrm{p}=0,886$ \\
\hline \multicolumn{8}{|l|}{ Resultados } \\
\hline Éxito global & 57 & $88 \%$ & 44 & $90 \%$ & 13 & $81 \%$ & $X^{2}, p=0,366$ \\
\hline Éxito funcional & 52 & $80 \%$ & 40 & $82 \%$ & 12 & $75 \%$ & \\
\hline Éxito anatómico & 5 & $8 \%$ & 4 & $8 \%$ & 1 & $6 \%$ & \\
\hline Fracaso & 8 & $12 \%$ & 5 & $10 \%$ & 3 & $19 \%$ & $X^{2}, p=0,657$ \\
\hline
\end{tabular}

ción bicanalicular, que habitualmente resultan dificultosas en las técnicas endoscópicas, pudieron realizarse sin complicación en todas las intervenciones. La sutura de las mucosas resultó complicada (sólo se pudo dar un punto) en 23 (35\%) de las intervenciones, sin diferencias entre adjuntos y residentes.

Como complicaciones postquirúrgicas valoramos la aparición de enfisema, epistaxis, reacción cicatricial queloide y la salida incompleta del tubo de silicona. No se produjo ningún enfisema, existieron 6 epistaxis, y sólo 4 reacciones queloides. Sin embargo hubo 13 (20\%) intervenciones en las que la salida del tubo de silicona no se produjo de forma completa, obligando a una exploración endoscópica.

Los resultados globales fueron de un $88 \%$ de éxito (80\% éxito funcional- $8 \%$ éxito anatómico), presentando el grupo de intervenciones de los adjuntos un $90 \%$ de éxito ( $82 \%$ funcional-8\% anatómico), mientras que el grupo de los residentes consiguió un $81 \%$ de éxito (75\% funcional-6\% anatómico). El porcentaje de fracasos global fue de un $12 \%$, siendo de un $10 \%$ para los adjuntos y de un $19 \%$ para los residentes, sin que se observaran diferencias estadísticas (tabla I).

En cuanto a las variables que se relacionaron con un mayor índice de fracasos sólo encontramos diferencias estadísticamente significativas $(\mathrm{p}=0,019)$ cuando se produjo la salida incompleta de los tubos de silicona (tabla II).

\section{DISCUSIÓN}

La DCREx continúa siendo la técnica quirúrgica más efectiva en el tratamiento de la OVLB en adultos. Ninguna técnica endoscópica (endonasal con tasas de éxito de 63-99\% o transcanalicular con láser con tasas de éxito de 50-85\%) ha conseguido la efectividad alcanzada con la DCREx que varía de 
Tabla II. Frecuencias observadas de las variables a estudio, según el resultado

\begin{tabular}{|c|c|c|c|c|c|c|c|}
\hline \multirow[b]{2}{*}{ Intervenciones DCREx (n) } & \multicolumn{2}{|c|}{$\begin{array}{c}\text { Éxito } \\
\text { Funcional }\end{array}$} & \multicolumn{2}{|c|}{$\begin{array}{c}\text { Éxito } \\
\text { Anatómico }\end{array}$} & \multicolumn{2}{|c|}{ Fracaso } & \multirow[t]{2}{*}{$\begin{array}{c}\text { Estadístico } \\
\text { y p-valor }\end{array}$} \\
\hline & 52 & $80 \%$ & 5 & $8 \%$ & 8 & $12 \%$ & \\
\hline Edad & \multicolumn{2}{|c|}{$65 \mathrm{DE} 11$} & \multicolumn{2}{|c|}{$67 \mathrm{DE} 12$} & \multicolumn{2}{|c|}{$73 \mathrm{DE} 7$} & $\begin{array}{c}\mathrm{T} \text { de Student } \\
\mathrm{p}=0,087\end{array}$ \\
\hline \multicolumn{8}{|l|}{ Sexo } \\
\hline Varones & 15 & $29 \%$ & & $40 \%$ & 3 & $38 \%$ & \\
\hline Mujeres & 37 & $71 \%$ & 3 & $60 \%$ & 5 & $63 \%$ & $X^{2}, p=0,794$ \\
\hline \multicolumn{8}{|l|}{ Lado } \\
\hline Derecho & 24 & $46 \%$ & 2 & $40 \%$ & 5 & $63 \%$ & \\
\hline Izquierdo & 28 & $54 \%$ & 3 & $60 \%$ & 3 & $38 \%$ & $X^{2}, p=0,647$ \\
\hline \multicolumn{8}{|l|}{ Inflamación } \\
\hline OVLB + Dacriocistitis & 17 & $33 \%$ & 1 & $20 \%$ & 4 & $50 \%$ & \\
\hline OVLB + Mucocele & 26 & $50 \%$ & 2 & $40 \%$ & 2 & $25 \%$ & \\
\hline OVLB + Simple & 10 & $19 \%$ & 2 & $40 \%$ & 2 & $25 \%$ & $X^{2}, p=0,559$ \\
\hline \multicolumn{8}{|l|}{ Anestesia } \\
\hline Local & 26 & $50 \%$ & 3 & $60 \%$ & 5 & $63 \%$ & \\
\hline General & 26 & $50 \%$ & 2 & $40 \%$ & 3 & $38 \%$ & $X^{2}, p=0,755$ \\
\hline \multicolumn{8}{|l|}{ Complicaciones intraquirúrgicas } \\
\hline Sangrado (Grado 1,2 ó 3) & 30 & $58 \%$ & 3 & $60 \%$ & 2 & $25 \%$ & $\mathrm{X}^{2}, \mathrm{p}=0,538$ \\
\hline Sangrado severo (Grado 3) & 6 & $12 \%$ & 0 & $0 \%$ & 1 & $13 \%$ & $\mathrm{X}^{2}, \mathrm{p}=0,719$ \\
\hline Trepanación defectuosa & 0 & $0 \%$ & 0 & $0 \%$ & 0 & $0 \%$ & \\
\hline Intubación defectuosa & 0 & $0 \%$ & 0 & $0 \%$ & 0 & $0 \%$ & \\
\hline Sutura mucosa 1 punto & 20 & $38 \%$ & 3 & $37 \%$ & 0 & $0 \%$ & $X^{2}, p=0,052$ \\
\hline \multicolumn{8}{|l|}{ Complicaciones postquirúrgicas } \\
\hline Enfisema & 0 & $0 \%$ & 0 & $0 \%$ & 0 & $0 \%$ & \\
\hline Epixtasis & 4 & $8 \%$ & 1 & $20 \%$ & 1 & $13 \%$ & $X^{2}, p=0,625$ \\
\hline Queloide & 2 & $4 \%$ & 1 & $20 \%$ & 1 & $13 \%$ & $\mathrm{X}^{2}, \mathrm{p}=0,260$ \\
\hline Salida incompleta tubos & 7 & $13 \%$ & 3 & $60 \%$ & 3 & $38 \%$ & $X^{2}, p=0,019$ \\
\hline \multicolumn{8}{|l|}{ Cirujano } \\
\hline Médico adjunto & 40 & $77 \%$ & 4 & $80 \%$ & 5 & $63 \%$ & \\
\hline Médico residente & 12 & $23 \%$ & 4 & $20 \%$ & 3 & $38 \%$ & $X^{2}, p=0,657$ \\
\hline
\end{tabular}

un $80-99 \%$ de éxito $(2,4)$. Podríamos ordenar por tasa de éxitos las distintas técnicas de dacriocistorrinostomía, resumiendo los resultados en: $70 \%$ de éxito en transcalicular con láser (5), 80\% en endonasal $(3,6)$ y $90 \%$ en DCREx $(1,7)$.

La DCREx presenta los mejores resultados ya que el saco lagrimal se visualiza directamente y esto permite eliminar las tabicaciones intrasacales, que se producen especialmente en situaciones de inflamación importante como en mucoceles o dacriocistitis, y suturar las mucosas nasal y sacular de un modo óptimo. Su principal desventaja es la cicatriz en el rostro que consideramos que puede ser disimulada en gran medida realizando la DCREx por vía inferior, empleando como vía de acceso el pliegue palpebral inferior (8).

Nuestros resultados ( $88 \%$ de éxito global) son comparables a los resultados medios de otros estudios. También hay que recordar que algunos de los estudios de DCREx con tasas de éxito más altas emplean antimitóticos (Mitomicina C) (9) que nosotros no hemos utilizado.
Respecto al objetivo del estudio hemos de concluir que en nuestra serie no se observan diferencias estadísticamente significativas entre las intervenciones realizadas por los médicos residentes y las realizadas por los médicos adjuntos, ni en cuanto a las complicaciones ni en cuanto a los resultados, aunque clínicamente los resultados son mejores en el grupo de intervenciones de los adjuntos $(90 \%$ de éxito) que en el de los residentes ( $81 \%$ de éxito). La única diferencia estadística en las intervenciones se observa en el empleo preferente de anestesia local por parte de los adjuntos y de anestesia general por parte de los residentes.

La única variable de las estudiadas que se relacionó estadísticamente con un mayor índice de fracaso fue la salida incompleta del tubo de silicona. La justificación de esta intubación es disminuir el riesgo de una obstrucción canalicular secundaria a las pequeñas laceraciones inducidas con las sondas de Bowman durante la manipulación de los canalículos y el saco lagrimal. Creemos que la salida del tubo de silicona valora de forma indirecta el grado 
de reacción cicatricial. La presencia de restos del tubo de silicona puede facilitar la aparición de un granuloma por cuerpo extraño que ocluya la vía lagrimal, por lo que aconsejamos insistir en la extracción completa de la sonda de silicona revisando por vía endoscópica la osteotomía o remitiendo al paciente al servicio de otorrinolaringología si no podemos conseguir la extracción completa del tubo. Respecto a la edad, aunque fue mayor en el grupo de pacientes con fracaso (73 años frente a 65 en el grupo con éxito) no resultó estadísticamente significativa.

Como conclusión, al igual que ocurre con otras intervenciones complicadas como son la cirugía cardiaca (10) o la tiroidea (11), las DCREx realizadas por médicos residentes, medidas en términos de complicaciones y resultados son similares a las realizadas por los cirujanos adjuntos por lo que esta técnica parece apta para ser realizada por médicos residentes bajo supervisión.

\section{BIBLIOGRAFÍA}

1. Boboridis $K G$, Bunce C, Rose GE. Outcome of external dacryocystorhinostomy combined with membranectomy of a distal canalicular obstruction. Am J Ophthalmol 2005; 139: 1051-1055.
2. Ayala-Barroso E, Medel-Jiménez R, Gonzalez-Candial M. Dacriocistorrinostomía: ¿externa, endonasal o endocanalicular? Arch Soc Canar Oftal 2006; 17: 100-105.

3. Küpper DS, Demarco RC, Resende R, Anselmo-Lima WT, Valera FC, Moribe I. Endoscopic nasal dacryocystorhinostomy: results and advantages over the external approach. Rev Bras Otorrinolaringol 2005; 71: 356-360.

4. Shun-Shin GA, Thurairajan G. External dacryocystorhinostomy - an end of an era? Br J Ophthalmol 1997; 81: 716-717

5. Mirza S, Al-Barmani A, Douglas SA, Bearn MA, Robson $A K$. A retrospective comparison of endonasal KTP laser dacryocystorhinostomy versus external dacryocystorhinostomy. Clin Otolaryngol Allied Sci 2002; 27: 347-351.

6. Cokkeser Y, Evereklioglu C, Er H. Comparative external versus endoscopic dacryocystorhinostomy: results in 115 patients (130 eyes). Otolaryngol Head Neck Surg 2000; 123: 488-491.

7. Delaney YM, Khooshabeh R. External dacryocystorhinostomy for the treatment of acquired partial nasolacrimal obstruction in adults. Br J Ophthalmol 2002; 86: 533-535.

8. Harris GJ, Sakol PJ, Beatty RL. Relaxed skin tension line incision for dacryocystorhinostomy. Am J Ophthalmol 1989; 108: 742-743.

9. Liao SL, Kao SC, Tseng JH, Chen MS, Hou PK. Results of intraoperative mitomycin C application in dacryocystorhinostomy. Br J Ophthalmol 2000; 84: 903-906.

10. Baskett RJ, Buth KJ, Legaré JF, Hassan A, Hancock-Friesen $C$, Hirsch GM, et al. Is it safe to train residents to perform cardiac surgery? Ann Thorac Surg 2002; 74: 1043-1049.

11. Manolidis S, Takashima M, Kirby M, Scarlett M. Thyroid surgery: a comparison of outcomes between experts and surgeons in training. Otolaryngol Head Neck Surg 2001; 125: $30-33$ 